\title{
BMJ Open Point-of-care ultrasound-guided regional anaesthesia in older ED patients with hip fractures: a study to test the feasibility of a training programme and time needed to complete nerve blocks by ED physicians after training
}

To cite: Lee JS, Bhandari T, Simard R, et al. Point-of-care ultrasound-guided regional anaesthesia in older ED patients with hip fractures: a study to test the feasibility of a training programme and time needed to complete nerve blocks by ED physicians after training. BMJ Open 2021;11:e047113. doi:10.1136/ bmjopen-2020-047113

- Prepublication history and additional supplemental material for this paper are available online. To view these files, please visit the journal online (http://dx.doi.org/10.1136/ bmjopen-2020-047113)

Received 07 January 2021 Accepted 18 June 2021

Check for updates

(c) Author(s) (or their employer(s)) 2021. Re-use permitted under CC BY-NC. No commercial re-use. See rights and permissions. Published by BMJ.

For numbered affiliations see end of article.

Correspondence to Dr Jacques Simon Lee; jacques.lee@sinaihealth.ca

\section{ABSTRACT}

Objectives Point-of-care ultrasound-guided regional anaesthesia (POCUS-GRA) provides safe, rapid analgesia for older people with hip fractures but is rarely performed in the emergency department (ED). Self-perceived inadequate training and time to perform POCUS-GRA are the two most important barriers. Our objective is to assess the feasibility of a proposed multicentre, steppedwedge cluster randomised clinical trial (RCT) to assess the impact of a knowledge-to-practice (KTP) intervention on delirium.

Design Open-label feasibility study.

Setting An academic tertiary care Canadian ED (annual visits 60 000).

Participants Emergency physicians working at least one ED shift per week, excluding those already performing POCUS-GRA more than four times per year.

Intervention A KTP intervention, including 2-hour structured training sessions with procedure bundle and email reminders.

Primary and secondary outcome measures The primary feasibility outcome is the proportion of eligible physicians that completed training and subsequently performed POCUS-GRA. Secondary outcome is the time needed to complete POCUS-GRA. We also test the feasibility of the enrolment, consent and randomisation processes for the future stepped-wedge cluster RCT (NCT02892968).

Results of 36 emergency physicians, 4 (12\%) were excluded or declined participation. All remaining 32 emergency physicians completed training and 31 subsequently treated at least one eligible patient. Collectively, 27/31 (87.1\%) performed 102 POCUS-GRA blocks (range 1-20 blocks per physician). The median (IQR) time to perform blocks was 15 (10-20) min, and
Strengths and limitations of this study

A strength of this feasibility study was that we specifically addressed barriers identified in a published survey of 100 emergency department (ED) physicians to performing point-of-care ultrasound-guided regional anaesthesia in the ED.

- Another strength of this paper was that we enrolled almost all eligible ED physicians at our centre, reducing selection bias and improving the generalisability of our results.

- A limitation of our study was missing data regarding time to perform nerve blocks.

- Another limitation of our study was that we report data only from a single centre.

reduction in pain was 6/10 (3-7) following POCUS-GRA. There were no reported complications.

Conclusion Our KTP intervention, consent process and randomisation were feasible. The time to perform POCUSGRA rarely exceeded $30 \mathrm{~min}$, Our findings reinforce the existing data on the safety and effectiveness of POCUSGRA, mitigate perceived barriers to more widespread adoption and demonstrate the feasibility of trialling this intervention for the proposed stepped-wedge cluster RCT. Trial registration number Clinicaltrials.gov \#02892968

\section{INTRODUCTION}

Discovering effective treatments does not ensure their routine adoption. ${ }^{1}{ }^{2}$ Metaanalyses summarising decades of research demonstrate that regional anaesthesia is preferred for hip fracture patients in terms 
of analgesic efficacy, safety and speed of onset. ${ }^{3-12}$ Early point-of-care ultrasound-guided regional anaesthesia (POCUS-GRA) is an example of an effective practice that is not widely used. ${ }^{12}$ Specifically, nine studies and three systematic review demonstrate the efficacy of regional anaesthesia used preoperatively. ${ }^{3-10} 13$ None reported an inability of emergency physicians to learn the technique. $^{57-101314}$

In addition, four randomised trials ${ }^{67915}$ and two cohort studies $^{16}{ }^{17}$ reported that regional anaesthesia could reduce delirium substantially, with a pooled OR of 0.36 (95\% CI: 0.17 to 0.74 ) for the four trials and 0.24 (95\% CI: 0.08 to 0.72 ) for the cohort studies. ${ }^{3-5}$ Despite its established efficacy and potential to reduce delirium, adoption of POCUS-GRA by emergency physicians remains low. Training in basic POCUS did not become a mandatory part of the emergency medicine curriculum in Canada until 2009, but there remains significant variability in POCUS curricula. $^{18}$ Typically, individual physicians with an interest in POCUS have had to seek expensive advanced training courses to learn POCUS-GRA. As a result, many emergency departments (EDs) include physicians with a wide variety of training and experience in the use of POCUS-GRA. ${ }^{19}$ We surveyed 100 Canadian emergency physicians and found that less than $5 \%$ regularly performed POCUS-GRA in hip fractures. ${ }^{12}$ The most commonly reported barriers were: (1) self-perceived inadequacy of training and (2) the perceived time to perform POCUS-GRA. The second barrier of perceived time could represent a significant barrier given ED crowding, a serious concern globally. ${ }^{20-23}$ Minimal data on the time needed to complete POCUS-GRA exist. One study reported an average of 5-10 min using a blind anatomical landmark technique in 18 procedures conducted by only two ED physicians with expertise in the procedure, potentially limiting the generalisability of these results. ${ }^{7}$ The addition of POCUS guidance improves efficacy of regional anaesthesia versus blind techniques, ${ }^{24}$ likely improves safety by allowing direct visualisation of neurovascular structures, but preparation of the ultrasound machine also likely increases the total procedure time.

The current feasibility study supports the conduct of a future pragmatic, multicentre stepped-wedge cluster randomised clinical trial (RCT). ${ }^{25}$ This future cluster RCT will assess the impact of our knowledge-to-practice (KTP) intervention on delirium in older people with hip fractures (clinicaltrials.gov \#02892968) by comparing the incidence of delirium among patients treated by ED physicians before and after they undergo the KTP intervention.

\section{METHODS}

\section{Objectives}

Our overall objective is to assess the feasibility of a proposed multicentre cluster RCT. To address the first feasibility barrier of self-perceived inadequacy of training identified in our previous survey, ${ }^{12}$ we developed a structured, KTP intervention designed to train all emergency physicians at our site to use POCUS-GRA, not just those with advanced ultrasound or procedural expertise..$^{71013}$

The primary feasibility outcome is the proportion of emergency physicians who completed standardised training and subsequently performed POCUS-GRA in eligible patients. Our secondary feasibility outcome is the time needed for ED physicians to perform POCUS-GRA after training. We also test the feasibility of elements of the protocol for our proposed cluster RCT, including the consent process and enrolment rates for physicians and patients.

We will randomly assign the order of physician training in our future stepped-wedge cluster RCT; however, significant logistics are required to schedule such training for an entire ED. Thus, we tested the feasibility of our randomisation scheme in the current study and report the proportion of physicians who were successfully scheduled. Finally, we report the safety and efficacy of POCUS-GRA after ED physicians received training.

\section{Design, setting and participants}

We conducted a prospective feasibility study ${ }^{26} 27$ at an academic tertiary care ED (annual visits 60 000) in Toronto, Ontario, Canada. Emergency physicians working fewer than four shifts per month and those who reported performing POCUS-GRA more than four times in the previous year were excluded.

We included patients who were aged 65 years and older with a hip fracture. We excluded those who were delirious on initial assessment using the Confusion Assessment Method. ${ }^{28}$ While this was not necessary to test the feasibility of our KTP intervention, it was important to estimate the patient enrolment rate for our future cluster RCT. Similarly, allergy to local anaesthetic, anticoagulant use, those unable to provide consent with no substitute decision maker, and those with minimal initial pain defined as $\leq 3 / 10$ at rest and with movement on a 10-point Numeric Rating Scale were also excluded. To test the feasibility of the proposed consent model for patients, trained research assistants approached patients for deferred consent after their pain had been controlled, typically the next day. To test our proposed stepped-wedge cluster randomisation scheme, we used a random number generator to assign all eligible participating emergency physicians to one of nine identical training sessions in the current feasibility study. The study coordinator then scheduled training sessions approximately every 2-3 months throughout the 22-month study period. We started scheduling training sessions 6 months prior to starting the study and took advantage of regular monthly education days to assist with scheduling.

\section{Description of KTP intervention}

The KTP intervention included an in-person, 2-hour training session, and deploying and maintaining a supply of pre-assembled kits in the ED (see online supplemental file 1 for a list of block kit contents). 
A Blue phantom ${ }^{\mathrm{TM}}$ trainer

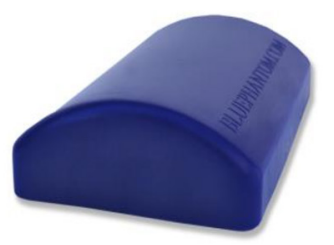

B Land marking on Live Patient Model

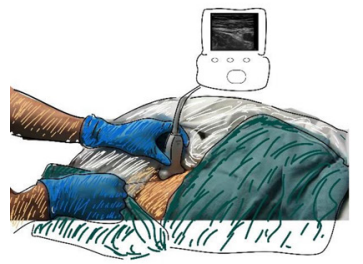

C Simulated Injection in Turkey Thigh Model (Image of Author)

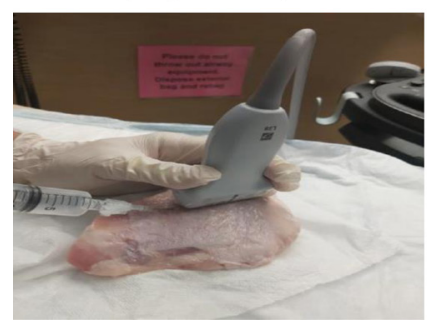

US appearance of Blue phantom ${ }^{\mathrm{TM}}$

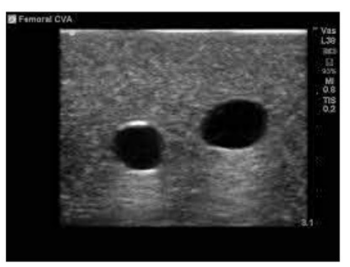

US appearance of Live Model Anatomy

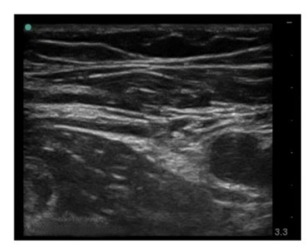

US appearance of Simulated Injection

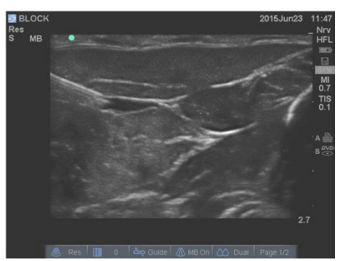

Figure 1 Illustration of three training stations used in 2hour training session. (A) Image of training station \#1 using Blue Phantom simulator station to practice finding femoral artery and vein and ultrasonographic appearance of the Blue Phantom. (B) Sketch of training station \#2 with proper placement of ultrasound machine and an example of the ultrasonographic appearance of a live model. (C) Image of training station \#3 showing injection in a turkey thigh model with the ultrasonographic appearance of injection in the model. US, ultrasound.

The standardised training sessions were led by three emergency physicians with fellowship training in POCUS and experience in performing and teaching POCUS-GRA (TB, RS and JC). Specifically, we taught the ultrasoundguided femoral nerve block procedure. In summary, the technique involves: (1) identifying the femoral nerve, artery, vein and the fascia iliaca plane using ultrasound. Both the nerve and the fascial plane appear as bright white structures on the ultrasound. (2) After anesthetising the skin, a 22-gauge spinal needle is introduced just lateral to the probe and advanced towards the femoral nerve until the needle crosses the fascia iliaca. (3) Next, $20 \mathrm{cc}$ of $0.5 \%$ bupivacaine with epinephrine is then injected under direct visualisation adjacent to the femoral nerve. Training sessions consist of a 30 - minute didactic session followed by practice on: (1) a phantom model mimicking femoral nerve anatomy (Blue Phantom, CAE Healthcare, Sarasota, Florida, USA); (2) a live model station for identification of anatomy and landmarks in an ageappropriate volunteer ( $\geq 70$ years); and (3) a turkey-thigh

station to practice dynamic needle guidance and ultrasonic visualisation of anaesthetic injection (figure 1). A video that supplemented training is available online (https://drive.google.com/open?id=0B3prgX0BFzo1czh VWnhWZk41a2c).

After demonstrating competence at all three stations and successfully completing a competency-based checklist as assessed by a study investigator (TB), each physician was encouraged to perform POCUS-GRA on all subsequent eligible hip fracture patients (see online supplemental file 2 for competency checklist). The procedure kits included laminated instructions plus all necessary equipment and medications, and were readily accessible in the ED day or night, and were restocked daily (see online supplemental file 3 for laminated instructions).

The final KTP strategy was reminders. Research staff reviewed the ED information system daily to identify consecutive eligible hip fracture patients and matched this list to data sheets completed by the ED physician to identify whether POCUS-GRA was performed. ${ }^{29}$ Research staff sent collegial email reminders to participating ED physicians if POCUS-GRA was not performed on eligible patients, and inquired as to the reason for not performing POCUS-GRA.

\section{Data collection and measurements}

Physicians were asked to record on a paper study form included in each procedure kit, to the nearest minute, the time when the kit was opened, and the time when the last step of the procedure was completed (applying a Band-Aid over the puncture site). They also recorded the patient's reported pain severity on a 10-point numeric rating scale at baseline and again $30 \mathrm{~min}$ postprocedure. Physicians were asked to record any complications from a prespecified list: minor (ie, local haematomas) or serious (femoral artery or nerve puncture, hypotension, seizures, shortness of breath, anaphylaxis or any other complication requiring treatment). Research assistants were also trained to review the medical record for any of these complications that were unreported (eg, comments on haematomas in the nursing record, administration of fluids, pressors or specialist consultations for a complication).

\section{Feasibility outcomes and targets}

The primary feasibility outcome was the proportion of emergency physicians who completed standardised training and subsequently performed at least one POCUS-GRA in eligible patients during the study. Completion of training was assessed using a competencybased checklist at the end of the 2-hour training session. Based on previous literature, we targeted $100 \%$ successful completion of training.

Our secondary feasibility outcome was the time needed for ED physicians to complete nerve blocks following training. We targeted $90 \%$ completion in under $40 \mathrm{~min}$ as feasible based on consensus among coinvestigators at our potential participating sites. 
Other secondary feasibility outcomes included assessing our consent process by measuring the proportion of physicians and patients who declined to participate. Feasibility of our scheme to randomise order of training was assessed by reporting the proportion of consenting physicians who could schedule and complete their randomly assigned training session.

We also wished to add to the existing evidence on the safety and efficacy of POCUS-GRA by reporting results among a more representative group of emergency physicians, not just those with advanced POCUS or procedural expertise. ${ }^{73}$ We used a reduction in pain score of $3 / 10$ as a minimally clinically important difference (MCID) based on previous literature, ${ }^{30}$ and targeted that $75 \%$ of patients would achieve this level of pain relief. Finally, we defined safety as the absence of any serious complications listed above.

\section{Sample size and statistical analysis}

Given that we were not testing any hypothesis, we based our sample size and statistical analysis for this prospective feasibility study on the precision or width of the $95 \%$ CI for our primary feasibility outcome, completion of training and subsequent POCUS-GRA use. Since it was possible that none would fail to complete training and subsequently perform a nerve block, we used the Hanley 'rule of three' to estimate the CI for proportions with a zero numerator. ${ }^{31}$ A sample of 30 physicians would yield a $95 \%$ CI of $0 \%$ to $10 \%$.

We report the proportion and 95\% CI for our primary feasibility outcome. For time to nerve block, we reported median, mean and IQR given the skewed distribution of time data.

\section{Patient and public involvement}

Patients were not involved in the planning and design of the current feasibility study, but were involved in the planning and design of our subsequent randomised trial.

\section{RESULTS}

\section{Demographics}

There were 52 attending emergency physicians with privileges during the 22-month study period. Seventeen worked casually (less than one shift per week on average) and were thus excluded. Of 36 eligible emergency physicians, $2(5.6 \%)$ routinely performed POCUS-GRA, and $2(5.6 \%)$ declined to participate. Participating physicians had an average of 12.4 years of experience and $68.8 \%$ were men (see table 1 for demographics).

\section{Primary feasibility outcome}

None of the 32 eligible physicians performed any POCUS-GRA procedures for hip fractures in the year prior to enrolment in this study. All 32 successfully completed a competency checklist following the 2-hour training session. One of the 32 emergency physicians was trained in the last 7 months of the study and did not assess

\begin{tabular}{|c|c|}
\hline Female physicians, n (\%) & $10(31.3)$ \\
\hline Experience in years, mean (IQR) & $12.4(11-22)$ \\
\hline Blocks performed (mean) & $102(3.13)$ \\
\hline Blocks with times recorded & 63 \\
\hline \multicolumn{2}{|l|}{ Time to block (min) } \\
\hline 25th percentile & 10.0 \\
\hline Median & 15.0 \\
\hline Mean & 15.5 \\
\hline 75th percentile & 22.0 \\
\hline 90th percentile & 30.0 \\
\hline
\end{tabular}

any eligible hip fracture patients from the time they were trained to the end of the study. Of the 31 trained physicians who did assess an eligible patient, $27 / 31$ (87.1\%; $95 \%$ CI: $70.2 \%$ to $96.4 \%$ ) performed at least one POCUS-GRA before the study ended, and 13/27 (48.1\%) performed three or more nerve blocks (see figure 2 for Consolidated Standards of Reporting Trials diagram of flow of patients into the study). The number of procedures performed by these physicians ranged from 0 to 20 (see figure 3 for completion rate of blocks by the physicians).

\section{Secondary feasibility outcomes: time to nerve block}

The time needed to perform POCUS-GRA was recorded by $22 / 27$ physician in $63 / 102$ cases $(61.8 \%)$. The median (IQR) time to complete nerve blocks was 15 (10-20) min, and $90 \%$ of blocks were completed in under $30 \mathrm{~min}$. There was significant variability in median time to block between physicians ranging from 4 to $35 \mathrm{~min}$ (see online supplemental table 1).

The impact of practice (ie, the number of nerve blocks performed) on time to perform blocks is presented in figure 4 . While there was a trend for reduced time to complete block with increasing number of procedures, this was not statistically significant. The Poisson regression model produced an incidence rate ratio of 0.98 (95\% CI: 0.95 to 1.02) for the impact of the number of nerve blocks performed on the time to complete the procedure. Of note, variability in time to complete blocks appeared to reduce after five blocks were performed, and 13/28 (46\%) ED physicians performed five or more blocks. For example, the median time to complete blocks if we include only first attempts at blocks $(n=25)$ was also $15 \mathrm{~min}$, and $90 \%$ were completed in $30 \mathrm{~min}$ (IQR 12.5$18.5 \mathrm{~min})$.

\section{Secondary feasibility outcome: physician consent rate and randomisation}

As noted above, 2/36 physicians declined participation $(5.6 \%)$.

We were able to schedule all remaining 32 emergency physicians to participate in the training session; they were randomised over 22 months. 


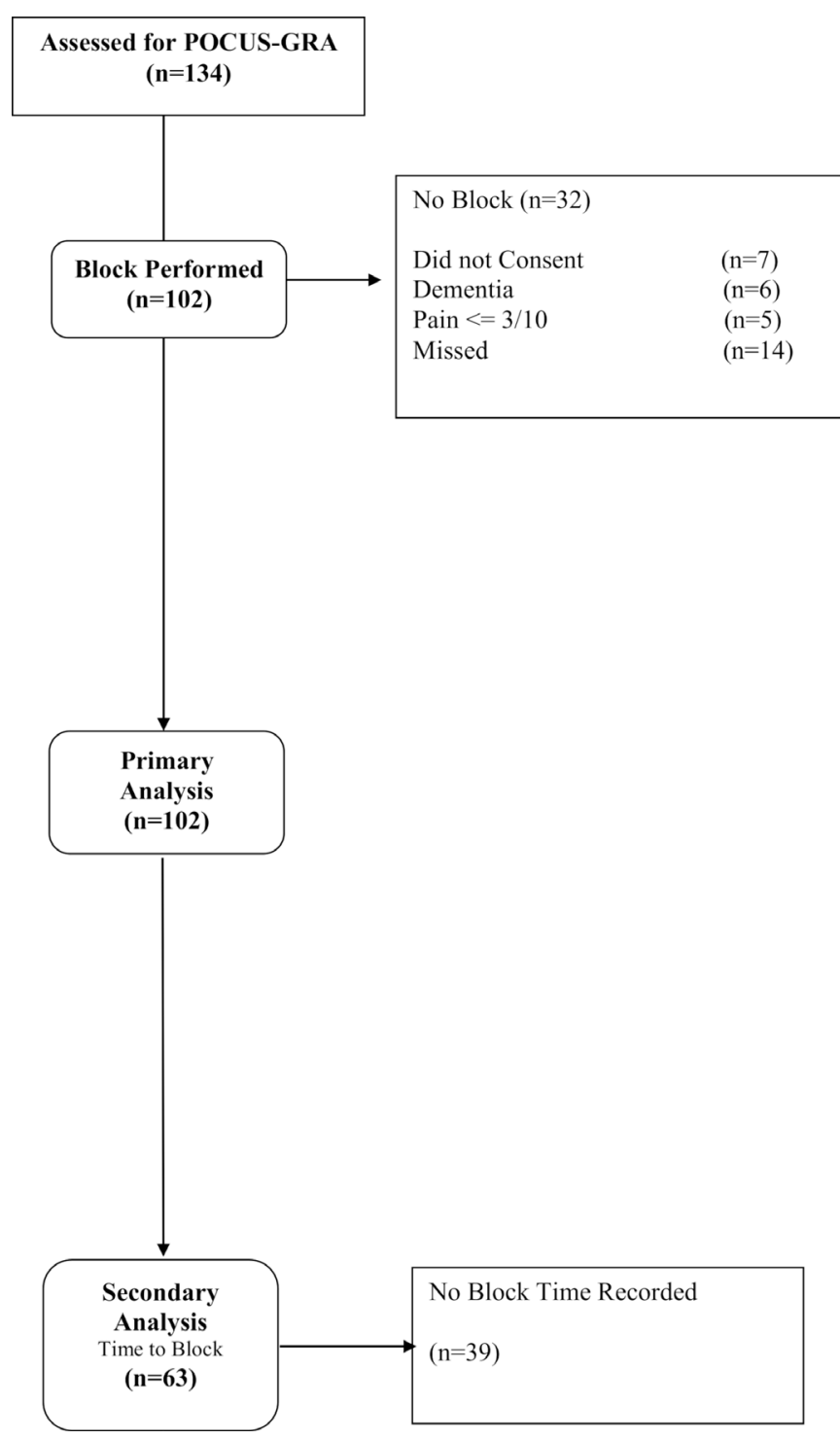

Figure 2 Consolidated Standards of Reporting Trials extension for feasibility studies. Diagram showing the flow of patients into the study. PROCUS-GRA, point-of-care ultrasound-guided regional anaesthesia.

\section{Secondary feasibility outcome: patient consent and enrolment rate}

Of the 134 hip fracture patients seen by the physicians who completed training, five had pain $\leq 3 / 10$ and were excluded. Another 7 declined to participate, 6 had dementia and 14 were missed. Altogether POCUS GRA was performed on 102/129 (79.1\%; 95\% CI: $71.3 \%$ to $85.7 \%$ ) eligible consecutive patients.

\section{Secondary feasibility outcome: efficacy and safety of nerve blocks}

Pain scores before nerve block and 30 min post block were recorded by the treating physician in 54/100 patients. Median (IQR) reduction in pain was 6 (3-7) on 10-point numeric rating scale. Using our MCID definition (see the Methods section) ${ }^{30} 51 / 55(94.4 \%)$ patients had a clinically important pain reduction of 3 points and 40/54 $(75 \%)$ patients had at least a $50 \%$ reduction in initial pain. No minor or serious complications were reported or identified on subsequent medical record review over the course of the study $(0 / 102,95 \%$ CI: $0 \%$ to $3.6 \%) .{ }^{31}$

\section{DISCUSSION}

This study evaluated the feasibility of a novel KTP intervention designed to train practising emergency physicians to use POCUS-GRA. The POCUS training was successfully delivered to $100 \%$ of emergency physicians, of which over $85 \%$ subsequently integrated it into their clinical practice and completed one or more POCUS-GRA in older patients who had sustained a hip fracture. In preparation for a future stepped-wedge cluster RCT, our feasibility study addressed the two most important barriers to performing POCUS-GRA that we had previously identified. ${ }^{12}$ Based on the success of our KTP intervention and the fact that most procedures could be completed in under $30 \mathrm{~min}$, we were able to recruit a network of seven EDs and over 200 emergency physicians from four provinces across Canada, obtain funding and launch a multicentre stepped-wedge RCT to assess the impact of our KTP intervention on delirium rates in older patients with hip fracture (clinicaltrials.gov \#02892968). The clinical trial finished follow-up in January 2021.

The strength of this feasibility study include the fact that we specifically addressed barriers to performing POCUS-GRA that had previously been identified by emergency physicians, then dealt with the training barrier by carefully designing and testing a multipronged KTP intervention that included hands-on simulation, competency assessment, an equipment bundle and reminders. Our study is the first to prospectively measure the time required to perform POCUS-GRA. Compared with previous studies that have used experts investigators exclusively, ${ }^{6-9}$ the fact that we enrolled $32 / 34$ (94\%) eligible physicians is another strength that reduces the probability of selection bias due to participation of early adopters or only physicians with exceptional POCUS skills. This improves the generalisability of our findings, and has implications for subsequent adoption of POCUS-GRA broadly into clinical practice. Limitations of this study include the fact that it was conducted at a single centre that was urban and academically affiliated, which may impact the generalisability of the results. We did feel it was important to establish feasibility at our centre before attempting implementation in a more challenging or resource-limited setting. But replicating our research in other settings will be important. Although the results may not extend to younger ( $<65$ years) adults, who were not included in this study, it is unlikely that time to complete the block will be longer for younger adults with anatomic landmarks that are easier to identify. Another limitation is the fact that we asked emergency physicians to record the time of their own block for pragmatic reasons, and that $1 / 3$ of times were missing. While it is possible that emergency physicians may have selectively failed to record the time of blocks that were more 


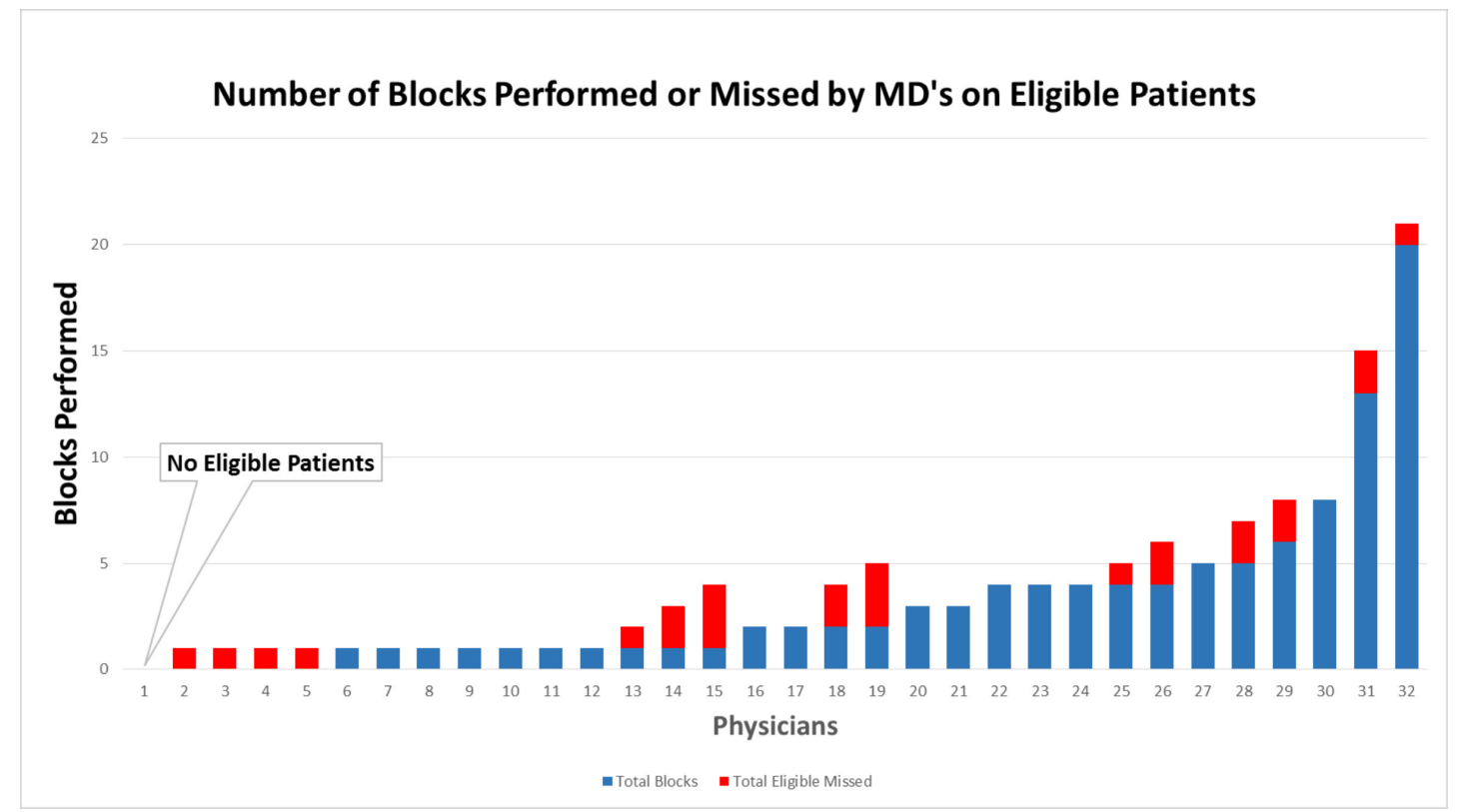

Figure 3 Bar graph illustrating the number of eligible patients assessed by all 32 participating physicians and the number blocked or missed.

challenging and took longer, ED physicians did record a wide variety of block times. Missing data on pain relief are another limitation; however, the effectiveness of regional anaesthesia for the pain of hip fractures has already been well established by several systematic reviews and two meta-analyses. ${ }^{3-5}$ Finally, only $48 \%$ of all participating physicians performing three or more blocks, generally considered as a minimum requirement to demonstrate competence in a procedure. However, because we tested the feasibility of randomising the order of training in this study, only $17 / 32(53.1 \%)$ saw three or more eligible patients prior to the end of the study. Of these, 13/17 $(76.5 \%)$ performed three or more blocks.

Our data agree with the previous literature and confirm the feasibility of training emergency physicians to perform POCUS-GRA, and that the procedure appears to be effective in controlling pain. ${ }^{7} 1013142432-35$ We observed no complications in 102 procedures, adding to the evidence

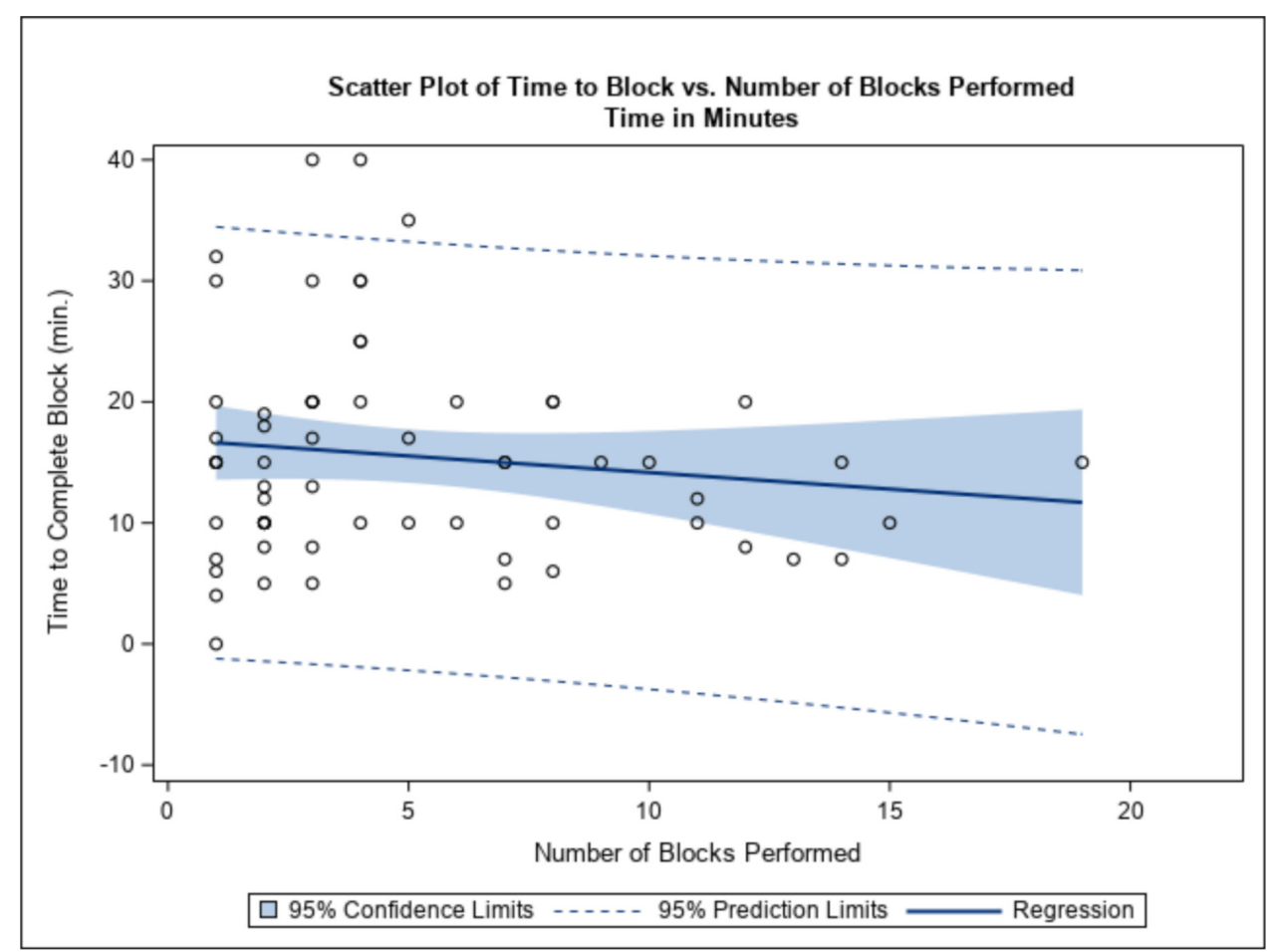

Figure 4 Scatter plot illustrating the relationship between the number of blocks performed ( $\mathrm{x}$-axis) and the time to complete blocks (y-axis) showing a reduction in variability of time to complete the blocks after five have been completed. 
that this procedure is safe even when performed by physicians who recently completed POCUS-GRA training. The fact that we excluded ultrasound experts and that $32 / 34$ eligible physicians participated in the study strengthens our confidence that these safety results are generalisable to other academic EDs.

The results of this study have implications for educators, researchers, clinicians and decision-makers. From an educational perspective, our results support the feasibility of our KTP programme to teach non-expert ED physicians to perform POCUS-GRA. With respect to research, establishing the median time required to complete POCUS-GRA was $15 \mathrm{~min}$ was instrumental in helping to recruit sites and physicians to participate in the trial and led to funding of a pragmatic multicentre steppedwedge cluster RCT to assess the impact of POCUS-GRA on delirium in older people with hip fractures.

Our safety, efficacy and feasibility results add to existing data and thus have implications for individual clinicians considering whether to learn the procedure to improve the analgesic care of older patients with a hip fracture. Our results show that practicing emergency physician who are not POCUS experts are able to complete nerve blocks after a 2-hour training session. For decision makers interested in increasing POCUS-GRA use above the current rate of $5 \%$ in the ED, ${ }^{12}$ our KTP programme provides a blueprint for implementation and provides data on potential uptake and impact on patient flow. Centres introducing POCUS-GRA may expect that a minority of physicians may need additional support while acquiring mastery over this procedure. It is of note that we carefully designed and tested an equipment bundle, which is a common quality improvement strategy to assist clinicians to adopt best practices and reduce the time required to perform blocks. Clearly, sites that do not choose to allocate resources to provide a procedure kit would likely experience significantly longer times to complete POCUS-GRA. We recognise that some individual physicians and institutions may consider the time required to perform POCUS-GRA as detracting from other tasks, especially when compared with the existing practice of simply paging orthopaedics and writing an order for parenteral opioids. While POCUS-GRA provides quicker, safer and more effective pain control compared with parenteral opioid analgesics, and has an important potential downstream effect of reducing inpatient delirium, its incorporation into routine clinical practice may be challenging because the existing alternative is highly time-efficient for emergency physicians facing multiple competing priorities and interruptions. Pain and discomfort in older patients with hip fractures have been shown to be adversely impacted by longer ED wait times. ${ }^{22}$

Future research should explore the reproducibility of our findings in other settings, assess sustainability of POCUS-GRA use over longer time frames and address the downstream impact of use of POCUS-GRA, including the potential to reduce delirium, hospital length of stay and ED crowding.

\section{CONCLUSION}

This study demonstrated the feasibility of our knowledge to practice programme, and the consent process and randomisation scheme for our proposed cluster RCT. Following a standardised 2-hour training programme along with email reminders and a prepackaged procedure kit, almost all trained emergency physicians performed POCUS-GRA on the majority of eligible hip fracture patients seen, and one-half performed three or more blocks. Our estimates of time to perform POCUS-GRA of 15 min suggest that time should not be a barrier to our trial nor to optimising analgesia for older people in the ED immediately following hip fracture. Our results add to existing data ${ }^{7} 1013$ that POCUS-GRA performed by ED physicians is safe and effective.

\section{Author affiliations}

${ }^{1}$ Schwartz/Reisman Emergency Medicine Institute, Sinai Health System, Toronto, Ontario, Canada

${ }^{2}$ Department of Medicine, University of Toronto, Toronto, Ontario, Canada

${ }^{3}$ Department of Emergency Services, Sunnybrook Health Sciences Centre, Toronto, Ontario, Canada

${ }^{4}$ Axe Santé des populations et pratiques optimales en santé, Universite Laval, Quebec, Québec, Canada

${ }^{5}$ Departément de medécine d'urgence, Universite Laval, Quebec, Québec, Canada ${ }^{6}$ Department of Family and Emergency Medicine, Universite Laval, Quebec, Québec, Canada

${ }^{7}$ Department of Emergency Medicine, University of Ottawa, Ottawa, Ontario, Canada ${ }^{8}$ Department of Emergency Medicine, Ottawa Hospital, Ottawa, Ontario, Canada ${ }^{9}$ Emergency Medicine, University of Calgary, Calgary, Alberta, Canada

${ }^{10}$ Department of Emergency Medicine, Queen's University, Kingston, Ontario, Canada

${ }^{11}$ Department of Family and Community Medicine, University of Toronto, Toronto, Ontario, Canada

${ }^{12}$ Department of Research Design and Biostatistics, Sunnybrook Research Institute, Toronto, Ontario, Canada

${ }^{13}$ Division of Emergency Medicine, University of Toronto Faculty of Medicine, Toronto, Ontario, Canada

Contributors JSL is the principal author, had full access to all of the data in the study and takes responsibility for the integrity of the data and the accuracy of the data analysis. He conceived and designed the study, obtained research funding, supervised the conduct of the data collection, prepared the manuscript and is responsible for the paper overall. MÉ, JP, MS and JC also contributed to the conception and obtaining funding for the study. TB, RS, JC, MÉ, CT, JP, DE, MW, $\mathrm{ADM}, \mathrm{EL}, \mathrm{CW}, \mathrm{MS}, \mathrm{JN}, \mathrm{BB}, \mathrm{SLM}, \mathrm{DM}$ and LC substantially contributed to the design, data collection, interpretation of data, and manuscript drafting and revision. AK substantially contributed to the design, analysis, interpretation and revision of the manuscript. All authors approved the final version of the manuscript.

Funding This work was supported by the Ontario Ministry of Health Innovation Fund (03212013).

Competing interests None declared.

Patient consent for publication Not required.

Ethics approval This study was approved by the Research Ethics Board at Sunnybrook Health Science Center (182-2012). All participating physicians provided written informed consent and patients provided delayed written informed consent.

Provenance and peer review Not commissioned; externally peer-reviewed.

Data availability statement № data are available.

Supplemental material This content has been supplied by the author(s). It has not been vetted by BMJ Publishing Group Limited (BMJ) and may not have been peer-reviewed. Any opinions or recommendations discussed are solely those of the author(s) and are not endorsed by BMJ. BMJ disclaims all liability and responsibility arising from any reliance placed on the content. Where the content includes any translated material, BMJ does not warrant the accuracy and reliability 
of the translations (including but not limited to local regulations, clinical guidelines, terminology, drug names and drug dosages), and is not responsible for any error and/or omissions arising from translation and adaptation or otherwise.

Open access This is an open access article distributed in accordance with the Creative Commons Attribution Non Commercial (CC BY-NC 4.0) license, which permits others to distribute, remix, adapt, build upon this work non-commercially, and license their derivative works on different terms, provided the original work is properly cited, appropriate credit is given, any changes made indicated, and the use is non-commercial. See: http://creativecommons.org/licenses/by-nc/4.0/.

\section{ORCID iDs}

Jacques Simon Lee http://orcid.org/0000-0002-9143-6285

Eddy Lang http://orcid.org/0000-0003-0850-4337

\section{REFERENCES}

1 Davis D, O'Brien MA, Freemantle N, et al. Impact of formal continuing medical education: do conferences, workshops, rounds, and other traditional continuing education activities change physician behavior or health care outcomes? JAMA 1999;282:867-74.

2 Graham ID, Tetroe J, KT Theories Research Group. Some theoretical underpinnings of knowledge translation. Acad Emerg Med 2007; 14:936-41.

3 Abou-Setta A, Beaupre L, Jones C. Pain management interventions for elderly patients with hip fracture. Rockville, MD: Agency for Healthcare Research and Quality, 2011.

4 Abou-Setta AM, Beaupre LA, Rashiq S, et al. Comparative effectiveness of pain management interventions for hip fracture: a systematic review. Ann Intern Med 2011;155:234-45.

5 Ritcey B, Pageau P, Woo MY, et al. Regional nerve blocks for hip and femoral neck fractures in the emergency department: a systematic review. CJEM 2016;18:37-47.

6 Foss NB, Kristensen BB, Bundgaard M, et al. Fascia iliaca compartment blockade for acute pain control in hip fracture patients: a randomized, placebo-controlled trial. Anesthesiology 2007;106:773-8.

7 Graham CA, Baird K, McGuffie AC. A pilot randomised clinical trial of 3-In-1 femoral nerve block and intravenous morphine as primary analgesia for patients presenting to the emergency department with fractured hip. Hong Kong J Emerg Med 2008;15:205-11.

8 Godoy Monzón D, Vazquez J, Jauregui JR, et al. Pain treatment in post-traumatic hip fracture in the elderly: regional block vs. systemic non-steroidal analgesics. Int J Emerg Med 2010;3:321-5.

9 Mouzopoulos G, Vasiliadis G, Lasanianos N, et al. Fascia iliaca block prophylaxis for hip fracture patients at risk for delirium: a randomized placebo-controlled study. J Orthop Traumatol 2009;10:127-33.

10 Beaudoin FL, Haran JP, Liebmann O. A comparison of ultrasoundguided three-in-one femoral nerve block versus parenteral opioids alone for analgesia in emergency department patients with hip fractures: a randomized controlled trial. Acad Emerg Med 2013;20:584-91.

11 Ontario $\mathrm{HQ}$. Care for people with fragility fractures: multimodal analgesia, 2021. Available: www.hqontario.ca/Evidence-to-ImproveCare/Quality-Standards/View-all-Quality-Standards/Hip-Fracture/ Quality-Statement-3-Multimodal-Analgesia [Accessed 27 Apr 2021].

12 Haslam L, Lansdown A, Lee J, et al. Survey of current practices: peripheral nerve block utilization by ED physicians for treatment of pain in the hip fracture patient population. Can Geriatr $J$ 2013;16:16-21

13 Beaudoin FL, Nagdev A, Merchant RC, et al. Ultrasound-Guided femoral nerve blocks in elderly patients with hip fractures. Am J Emerg Med 2010;28:76-81.

14 Fletcher AK, Rigby AS, Heyes FLP. Three-in-one femoral nerve block as analgesia for fractured neck of femur in the emergency department: a randomized, controlled trial. Ann Emerg Med 2003;41:227-33.

15 Godoy Monzon D, Iserson KV, Vazquez JA. Single fascia iliaca compartment block for post-hip fracture pain relief. J Emerg Med 2007;32:257-62.

16 Griffith JP, Whiteley S, Gough MJ. Prospective randomized study of a new method of providing postoperative pain relief following femoropopliteal bypass. Br J Surg 1996;83:1735-8.

17 Guryay D, Karaege GT, Katircioglu K, et al. The effects of an epidural infusion of ropivacaine versus saline on sensory block after spinal anesthesia. Reg Anesth Pain Med 2008;33:217-21.

18 Moore CL, Gregg S, Lambert M. Performance, training, quality assurance, and reimbursement of emergency physician-performed ultrasonography at academic medical centers. J Ultrasound Med 2004;23:459-66.

19 Amini R, Kartchner JZ, Nagdev A, et al. Ultrasound-Guided nerve blocks in emergency medicine practice. $J$ Ultrasound Med 2016;35:731-6.

20 Pines JM, Mullins PM, Cooper JK, et al. National trends in emergency department use, care patterns, and quality of care of older adults in the United States. J Am Geriatr Soc 2013;61:12-17.

21 Rowe BH, Bond K, Ospina M. Emergency department overcrowding in Canada: what are the issues and what can be done? Technical overview No. 21. Ottawa Canadian Agency for Dructs and Technologies in Health; 2006.

22 Hwang U, Richardson LD, Sonuyi TO, et al. The effect of emergency department crowding on the management of pain in older adults with hip fracture. J Am Geriatr Soc 2006;54:270-5.

23 Richardson DB, Mountain D. Myths versus facts in emergency department overcrowding and hospital access block. Med J Aust 2009;190:369-74.

24 Reid N, Stella J, Ryan M, et al. Use of ultrasound to facilitate accurate femoral nerve block in the emergency department. Emerg Med Australas 2009;21:124-30.

25 Hussey MA, Hughes JP. Design and analysis of stepped wedge cluster randomized trials. Contemp Clin Trials 2007;28:182-91.

26 Eldridge SM, Chan CL, Campbell MJ, et al. CONSORT 2010 statement: extension to randomised pilot and feasibility trials. BMJ 2016;355:i5239.

27 Eldridge SM, Lancaster GA, Campbell MJ, et al. Defining feasibility and pilot studies in preparation for randomised controlled trials: development of a conceptual framework. PLoS One 2016;11:e0150205.

28 Inouye SK, van Dyck CH, Alessi CA, et al. Clarifying confusion: the confusion assessment method. A new method for detection of delirium. Ann Intern Med 1990;113:941-8.

29 Verma A, Wang AS, Feldman MJ, et al. Push-Alert Notification of Troponin Results to Physician Smartphones Reduces the Time to Discharge Emergency Department Patients: A Randomized Controlled Trial. Ann Emerg Med 2017;70:348-56.

30 Lee JS, Hobden E, Stiell IG, et al. Clinically important change in the visual analog scale after adequate pain control. Acad Emerg Med 2003;10:1128-30.

31 Hanley JA, Lippman-Hand A. If nothing goes wrong, is everything all right? Interpreting zero numerators. JAMA 1983;249:1743-5.

32 Finlayson BJ, Underhill TJ. Femoral nerve block for analgesia in fractures of the femoral neck. Arch Emerg Med 1988;5:173-6.

33 Van Leeuwen FL, Bronselaer K, Gilles M, et al. The 'three in one' block as locoregional analgesia in an emergency department. Eur J Emerg Med 2000;7:35-8.

34 Mutty CE, Jensen EJ, Manka MA, et al. Femoral nerve block for diaphyseal and distal femoral fractures in the emergency department. J Bone Joint Surg Am 2007;89:2599-603.

35 Rogers BA, Rang S. Femoral nerve block for diaphyseal and distal femoral fractures in the emergency department. J Bone Joint Surg Am 2008;90:1787-8. 